\title{
Four-valued Extension of Rough Sets ${ }^{\star}$
}

\author{
Aida Vitória ${ }^{1}$, Andrzej Szałas ${ }^{2,3}$, and Jan Małuszyński ${ }^{3}$ \\ ${ }^{1}$ Department of Science and Technology, Linköping University \\ S 60174 Norrköping, Sweden, aidvi@itn.liu.se \\ ${ }^{2}$ Institute of Informatics, Warsaw University \\ 02-097 Warsaw, Poland, andsz@mimuw.eu.pl \\ 3 Department of Computer and Information Science, Linköping University \\ 58183 Linköping, Sweden, janma@ida.liu.se
}

\begin{abstract}
Rough set approximations of Pawlak [15] are sometimes generalized by using similarities between objects rather than elementary sets. In practical applications, both knowledge about properties of objects and knowledge of similarity between objects can be incomplete and inconsistent. The aim of this paper is to define set approximations when all sets, and their approximations, as well as similarity relations are four-valued. A set is four-valued in the sense that its membership function can have one of the four logical values: unknown $(\mathbf{u})$, false (f), inconsistent (i), or true (t). To this end, a new implication operator and set-theoretical operations on four-valued sets, such as set containment, are introduced. Several properties of lower and upper approximations of four-valued sets are also presented.
\end{abstract}

\section{Introduction}

Rough sets [15] are constructed by means of approximations obtained by using elementary sets which partition a universe. The assumption as to partitioning of the universe has been relaxed by many authors (see, e.g., [1, 6, 9, 17-19]), however the Pawlak's idea of approximations has remained the same. Namely, an object $o$ belongs to the lower approximation of a given set $A$ whenever all objects indiscernible from $o$ belong to $A$ and $o$ belongs to the upper approximation, when there are objects indiscernible from $o$ belonging to $A$. Indiscernibility is modeled by similarity relations reflecting limited perceptual capabilities as well as incomplete and imprecise knowledge. Such approximations naturally lead to three- and four-valued logics (see, e.g., [3, 8, 10, 14]).

The goal of the current paper is to ground Pawlak's ideas in a four-valued framework. To this end, we define a four-valued set theory in the sense that the membership function, set containment as well as union, intersection and complement of sets are four-valued. We may then have that either an element belongs to a given set, or it does not belong to the set, or its membership in the set may be unknown or inconsistent, perhaps, due to contradictory evidence. Notice that we assume that all sets and relations are four-valued, in particular the underlying similarity relation. To our knowledge, this is a fundamental difference between the work we present and the work discussed by other authors in the rough-set field. Therefore, we take into account the fact that in practical applications our knowledge about similarity between objects of the universe can also be incomplete and inconsistent. This novel aspect of our work has led us to re-think and extend the usual notions of upper and lower approximations. Since we consider similarity relations as four-valued sets, there are cases, when we cannot establish with certainty whether the neighborhood of an element is a subset of (or whether is disjoint with) a given set. To

\footnotetext{
* Supported in part by the MNiSW grant N N206 399334 and by the 6th Framework Programme project REWERSE number 506779 (cf. http://rewerse.net).
} 
tackle this problem, we propose upper and lower approximations that are also fourvalued sets. It is important to note that in cases, when only the standard truth values $\mathbf{t}$ and $\mathbf{f}$ are used, all notions we define reduce to the standard operations on rough sets.

The work presented in [11] also captures the same type of knowledge uncertainty, as described above. However, uncertainty in the properties of objects is captured by fuzzy sets and (two-valued) equivalence relations are used to capture similarity between objects of the universe. Upper and lower approximation of a fuzzy set are defined in [11]. Unlike [11], in our framework, similarity between objects is captured by four-valued relations and all properties correspond to four-valued sets.

When considering similarities as well as approximations to be four-valued, as we do in the current paper, it appears that the truth ordering proposed in [14] makes definitions of set containment and approximations problematic. Therefore, we propose to slightly modify the truth ordering (by changing the relationship between $\mathbf{f}$ and $\mathbf{u}$ only).

The paper is structured as follows. First, in Section 2, we formalize the notion of fourvalued set. Section 3 is devoted to four-valued approximations. In Section 4, we provide an example illustrating the approach. Finally, Section 5 concludes the paper.

\section{The Framework}

In this section, we first define four-valued sets together with the notions of four-valued set containment and four-valued set intersection and union. To formalize four-valued set containment, we introduce a new implication connective.

\subsection{Four-valued Sets}

Let $\mathcal{B}=\{\mathbf{t}, \mathbf{f}, \mathbf{i}, \mathbf{u}\}$ be the set of truth values, where $\mathbf{t}$ stands for true, $\mathbf{f}$ stands for false, $\mathbf{i}$ stands for inconsistent and $\mathbf{u}$ stands for unknown. Any of these logical values can be negated: $\neg \mathbf{t}=\mathbf{f}, \neg \mathbf{i}=\mathbf{i}, \neg \mathbf{u}=\mathbf{u}$, and $\neg \mathbf{f}=\mathbf{t}$.

Let us now formalize the notion of four-valued sets. Given a universe $U$, we introduce a new set, disjoint with $U$, denoted by $\neg U$ and defined by $\neg U \stackrel{\text { def }}{=}\{\neg x \mid x \in U\}$, where $\neg x$ denotes elements in $\neg U$. Intuitively, $x \in A$ represents the fact that there is an evidence that $x$ is in $A$ and $(\neg x) \in A$ represents the fact that there is an evidence that $x$ is not in $A$. A four-valued set $A$ on $U$ is any subset of $U \cup \neg U$.

In our framework, set membership is four-valued and it extends the usual two-valued membership. We assume that $\neg(\neg x)$ is equivalent to $x$.

Definition 1. Set membership, denoted as $\epsilon: U \times 2^{U \cup \neg U} \rightarrow \mathcal{B}$, is defined by:

$$
x \in A=\left\{\begin{array}{l}
\mathbf{t} \text { if } x \in A \text { and }(\neg x) \notin A \\
\mathbf{i} \text { if } x \in A \text { and }(\neg x) \in A \\
\mathbf{u} \text { if } x \notin A \text { and }(\neg x) \notin A \\
\mathbf{f} \text { if } x \notin A \text { and }(\neg x) \in A .
\end{array}\right.
$$

The complement $\neg A$ of a four-valued set $A$, is defined by $\neg A \stackrel{\text { def }}{=}\{\neg x \mid x \in A\}$.

Let $P$ be a four-valued set on a universe $U$. To simplify the notation, $P(x)$ stands for $x \in P$. For instance, let $U=\{a, b, c\}$ and $\operatorname{Red}=\{a, \neg a, b\}$, In this case, $\operatorname{Red}(b)=\mathbf{t}$ and $\operatorname{Red}(a)=\mathbf{i}$, since both $a \in \operatorname{Red}$ and $\neg a \in \operatorname{Red}$. Moreover, $\neg \operatorname{Red}=\{a, \neg a, \neg b\}$. 


\subsection{Four-valued Calculus}

In our work, we use two orderings of the logical values: truth ordering and knowledge ordering. Truth ordering is used for calculations within a single information source while knowledge ordering is used for gathering knowledge from different sources. This approach has been considered by in [4] and in the framework of bilattices, in [13, 12].

The truth ordering $\leq_{t}$ and the knowledge ordering $\leq_{k}$ on $\mathcal{B}$ are defined as the smallest reflexive and transitive relations satisfying $\mathbf{f} \leq_{t} \mathbf{u} \leq_{t} \mathbf{i} \leq_{t} \mathbf{t}, \mathbf{u} \leq_{k} \mathbf{f} \leq_{k} \mathbf{i}$, and $\mathbf{u} \leq_{k} \mathbf{t} \leq_{k} \mathbf{i}$. The disjunctions $\vee_{t}$ and $\vee_{k}$ (conjunctions $\wedge_{t}$ and $\wedge_{k}$ ) are defined to be the least upper bounds (greatest lower bounds) of their arguments w.r.t. $\leq_{t}$ and $\leq_{k}$, respectively.

Let us relate the orderings above with Belnap's truth and knowledge ordering [4]. The knowledge ordering we defined above coincides with Belnap's knowledge ordering. However, our truth ordering is different from the Belnap's truth ordering. This change is motivated by the fact that Belnap's truth ordering can give counterintuitive results when used for reasoning, as shown in [14].

We also need to extend the notion of set containment and set intersection to four-valued sets. We discuss next these two ideas.

Given two four-valued sets $A_{1}$ and $A_{2}$ over a universe $U, A_{1} \Subset A_{2}$ stands for $A_{1}$ being contained in $A_{2}$. Notice that our notion of set containment is also four-valued. Thus, $A_{1} \Subset A_{2}$ can be evaluated to one of the four logical values in $\mathcal{B}$. If $A$ and $B$ are twovalued sets, then set containment is defined as $A \subseteq B \stackrel{\text { def }}{=} \forall x \in U(x \in A \rightarrow x \in B)$. This definition relies on the notion of universal quantification and logical implication $\rightarrow$. We follow a similar idea. Therefore, we first extend the notion of universal and existential quantification. We then propose a new implication connective, $\hookrightarrow$, for our four-valued logic.

Since $\forall(\exists)$ is a generalized conjunction (a generalized disjunction), we define

$$
\forall x[P(x)] \stackrel{\text { def }}{=} \operatorname{GLB}_{x \in U}^{t}\{P(x)\} \text { and } \exists x[P(x)] \stackrel{\text { def }}{=} \underset{x \in U}{\operatorname{LuB}}{ }^{t}\{P(x)\}
$$

where the superscript ${ }^{t}$ indicates that the greatest lower bound (GLB) and least upper bound (LUB) are computed w.r.t. truth ordering.

We define now the semantics of a new implication operator $\hookrightarrow$. To define this operator, we must have in mind that it should be suitable for determining set containment, in the case of four-valued sets. Obviously, $\hookrightarrow$ should also be an extension of the usual logical implication $\rightarrow$. We present below a table defining $\hookrightarrow$ and then, we provide the motivation.

\begin{tabular}{c|c|c|c|c}
$\hookrightarrow$ & $\mathbf{f}$ & $\mathbf{u}$ & $\mathbf{i}$ & $\mathbf{t}$ \\
\hline $\mathbf{f}$ & $\mathbf{t}$ & $\mathbf{t}$ & $\mathbf{t}$ & $\mathbf{t}$ \\
$\mathbf{u}$ & $\mathbf{u}$ & $\mathbf{u}$ & $\mathbf{i}$ & $\mathbf{t}$ \\
$\mathbf{i}$ & $\mathbf{i}$ & $\mathbf{i}$ & $\mathbf{i}$ & $\mathbf{t}$ \\
$\mathbf{t}$ & $\mathbf{f}$ & $\mathbf{u}$ & $\mathbf{i}$ & $\mathbf{t}$ \\
\hline
\end{tabular}

Let $X$ and $Y$ be two four-valued sets and assume we want to verify whether $X \Subset Y$. If we know that an element $x$ does not belong to $X$ (i.e., $x \in X=\mathbf{f}$ ) then the membership 
of $x$ in $Y$ should have no influence on the result of $X \Subset Y$. This motivates the first line of the table. If $x$ belongs to $Y$ (i.e., $x \in Y=\mathbf{t}$ ) then the truth valued of $x \epsilon X$ is irrelevant. This explains the last column of the table above. If $x$ belongs to $X$ (i.e., $x \epsilon X=\mathbf{t}$ ) then our conclusions w.r.t. element $x$ should depend only on our knowledge about $x \in Y$. This is the intuition behind the last line of the table. It seems obvious that if we know nothing about membership of $x$ in $X$ and nothing about membership of $x$ in $Y$ (i.e., both $x \in X$ and $x \epsilon Y$ are evaluated to $\mathbf{u}$ ) then, we cannot conclude anything about the contribution element $x$ gives to determine whether $X$ is a subset of $Y$. Thus, $\mathbf{u} \hookrightarrow \mathbf{u}$ is evaluated to $\mathbf{u}$. Similarly, $\mathbf{i} \hookrightarrow \mathbf{i}$ is evaluated to $\mathbf{i}$.

Let us now discuss the remaining cases. If $\hookrightarrow$ is going to be used to determine set containment, then it is desirable that it has the following property: $\left(b_{1} \hookrightarrow b_{2}\right)=\left(\neg b_{2} \hookrightarrow\right.$ $\left.\neg b_{1}\right)$. Since $\mathbf{t} \hookrightarrow \mathbf{u}$ is evaluated to $\mathbf{u}$ and $\mathbf{t} \hookrightarrow \mathbf{i}$ is evaluated to $\mathbf{i}$, we should then have that $\mathbf{u} \hookrightarrow \mathbf{f}$ and $\mathbf{i} \hookrightarrow \mathbf{f}$ should be evaluated to $\mathbf{u}$ and $\mathbf{i}$, respectively.

If we have no information about whether $x$ belongs to $X$ (i.e., $x \in X=\mathbf{u}$ ) and we have contradictory evidence about the membership of $x$ in $Y$ (i.e., $x \in Y=\mathbf{i}$ ), then future information about membership of $x$ in $X$ can lead us to the conclusion that

- element $x$ contributes with $\mathbf{i}$, if $x \in X$ is evaluated to $\mathbf{t}$ (note that $\mathbf{t} \hookrightarrow \mathbf{i}=\mathbf{i}$ ), or

- element $x$ contributes with $\mathbf{t}$, if $x \in X$ is evaluated to $\mathbf{f}$ (note that $\mathbf{f} \hookrightarrow \mathbf{i}=\mathbf{t}$ ), or

- element $x$ contributes with $\mathbf{i}$, if $x \in X$ is evaluated to $\mathbf{i}$ (note that $\mathbf{i} \hookrightarrow \mathbf{i}=\mathbf{i}$ ).

Thus, we define $\mathbf{u} \hookrightarrow \mathbf{i}$ to be $\mathbf{i}$ since that is a possible value for the contribution of element $x$ in the future, when more knowledge is gathered, but it still conveys a degree of uncertainty. Moreover, we have then that $\mathbf{i} \hookrightarrow \mathbf{u}$ should also be defined as $\mathbf{i}$.

Other implication connectives have been proposed by other authors (see, e.g., [2]). Let us make a brief comparison. Obviously, the proposed implication $\hookrightarrow$ extends the usual two-valued logical implication, i.e. when we only consider the logical values $\mathbf{t}$ and $\mathbf{f}$. It also extends the implication of the Kleene three-valued logic $[5,16]$ in the sense that when we restrict truth values to $\{\mathbf{t}, \mathbf{f}, \mathbf{u}\}$ (or $\{\mathbf{t}, \mathbf{f}, \mathbf{i}\}$ ), we obtain Kleene's implication.

On the other hand, our implication differs from the material implication $\mapsto$ proposed in [2], on the following two cases: $\mathbf{u} \mapsto \mathbf{i}=\mathbf{t}$ while $\mathbf{u} \hookrightarrow \mathbf{i}=\mathbf{i}$ and $\mathbf{i} \mapsto \mathbf{u}=\mathbf{t}$ while $\mathbf{i} \hookrightarrow \mathbf{u}=\mathbf{i}$. The material implication $\mapsto$ can be defined by means of negation and disjunction. The same property turns out to be true for our implication connective, i.e. $\left(b_{1} \hookrightarrow b_{2}\right)=\left(\neg b_{1} \vee_{t} b_{2}\right)$. In contrast to the internal implication $\supset$ proposed in [2], our implication does not satisfy the Modus Ponens, if we assume that $\{\mathbf{t}, \mathbf{i}\}$ is the set of designated values. For instance, $\mathbf{i} \hookrightarrow \mathbf{f}=\mathbf{i}$.

Observe that given two four-valued sets $X$ and $Y$ over a universe $U$, we can define a new four-valued set $P$, such that $(x \in P)$ has the same truth value as $(x \in X \hookrightarrow x \in Y)$, for every object $x \in U$. We can now formally define set containment, for four-valued sets.

Definition 2. Assume that $X$ and $Y$ are four-valued sets on a universe $U$. Then,

$$
X \Subset Y \stackrel{\text { def }}{=} \forall x \in U(x \in X \hookrightarrow x \in Y)
$$

We say $X$ is a subset of $Y$ iff $X \Subset Y=\mathbf{t}$ and $X$ is not a subset of $Y$, denoted by $X \notin Y$, iff $X \Subset Y=\mathbf{f}$. 
The following proposition can be proved.

Proposition 1. Let $X$ and $Y$ be four-valued sets on a universe $U$. Then, $(X \Subset Y)=$ $(\neg Y \Subset \neg X)$.

Let us now define the notion of four-valued set intersection $\cap$ and union $\amalg$.

Definition 3. Let $X$ and $Y$ be four-valued sets on a universe $U$. Then,

$$
x \epsilon(X \cap Y) \stackrel{\text { def }}{=}(x \epsilon X) \wedge_{t}(x \in Y) \text { and } x \epsilon(X \uplus Y) \stackrel{\text { def }}{=}(x \epsilon X) \vee_{t}(x \epsilon Y) .
$$

Note that $\mathrm{n}$ and $\mathbb{e}$ reduce to the standard two-valued set intersection and union when only values $\mathbf{t}$ and $\mathbf{f}$ are present.

\section{Four-valued Set Approximations}

In the usual rough-set framework, given a set and a similarity relation, lower and upper approximations of the set can be obtained. We extend these ideas to four-valued sets. In contrast to previous work, we deal with four-valued sets on a given universe and fourvalued similarity relations. Moreover, upper and lower approximations of four-valued sets are themselves four-valued sets.

Definition 4. By a four-valued similarity relation $\sigma$ we mean any four-valued binary relation on a universe $U$, satisfying the reflexivity condition, i.e., for any element $x$ of the universe $(x, x) \epsilon \sigma=\mathbf{t}$. By the neighborhood of element $x \in U$ w.r.t. $\sigma$, we understand the four-valued set $\sigma(x)$ such that $y \in \sigma(x) \stackrel{\text { def }}{=}(x, y) \epsilon \sigma$.

We proceed now with the definitions of four-valued approximations.

Definition 5. Let $A$ be a four-valued set. Then, the lower and upper approximations of $A$ w.r.t. $\sigma$, denoted by $A_{\sigma}^{+}$and $A_{\sigma}^{\oplus}$, respectively, are defined by $x \in A_{\sigma}^{+} \stackrel{\text { def }}{=} \sigma(x) \Subset A$ and $x \in A_{\sigma}^{\oplus} \stackrel{\text { def }}{=} \exists y \in U[y \in(\sigma(x) \cap A)]$.

The definitions above naturally extend the usual definitions of lower and upper approximations presented in the rough set literature. We determine the membership of an element $x$ in the lower approximation by verifying (set) containment of its neighborhood, $\sigma(x)$, in set $A$. We determine the membership of an element $x$ in the upper approximation by computing the largest membership value that an element of the universe can have in the intersection of $\sigma(x)$ and $A$, w.r.t. to $\leq_{t}$.

The next theorems summarize some important properties of lower and upper approximations of four-valued sets.

Theorem 1. Let $A$ be a four-valued set on a universe $U, \sigma$ be a four-valued similarity relation. Then, $\left(x \in A_{\sigma}^{+}\right) \leq_{t}\left(x \in A_{\sigma}^{\oplus}\right)$, for all $x \in U$. 
The above theorem lead us to the conclusion that it is never the case that $A_{\sigma}^{+} \notin A_{\sigma}^{\oplus}$ and it is formalized below. From an intuitive point of view, this conclusion is the counterpart of a known property of rough-set approximations: the lower approximation is always contained in the upper approximation, when usual (two-valued) sets are used.

Theorem 2. Let $A$ be a four-valued set on a universe $U, \sigma$ be a four-valued similarity relation. Then, $\left(A_{\sigma}^{+} \Subset A_{\sigma}^{\oplus}\right)>_{t} \mathbf{f}$.

\section{An Example}

In this section, we present an example that illustrates the discussed ideas.

Perception can be modelled by similarity relations in the sense that objects indiscernible due to the perceptive limitations are considered similar to each other (see, e.g., [7], where incomplete knowledge about similarities has been taken into account in the context of perception). However, in our framework, in addition to incompleteness, the knowledge of similarity between objects can also be inconsistent. For instance, two different sensors, may give contradictory evidence about the similarity of two objects. The universe $U=\{a, b, c, d\}$ consists of objects classified as being dangerous. The four-valued set Danger represents this property. Note that this classification may be incomplete in some cases and uncertain in others. For instance, for object $d$ there is no information about its danger, while for for object $c$ there is contradictory evidence about whether it is dangerous (i.e. $c \in$ Danger $=\mathbf{i}$ ).

Suppose that we have four information sources, denoted by $I_{i}(i \in\{1,2,3,4\})$, about the objects similarity, modelled by the similarity relations $\sigma_{i}$, respectively.

The set Danger and the similarities of $a$ to other elements of the universe, $\sigma_{i}(a)$, are given below.

\begin{tabular}{c|c|c|c|c} 
& $a$ & $b$ & $c$ & $d$ \\
\hline$\sigma_{1}(a)$ & $\mathbf{t}$ & $\mathbf{i}$ & $\mathbf{u}$ & $\mathbf{f}$ \\
$\sigma_{2}(a)$ & $\mathbf{t}$ & $\mathbf{f}$ & $\mathbf{f}$ & $\mathbf{f}$ \\
$\sigma_{3}(a)$ & $\mathbf{t}$ & $\mathbf{t}$ & $\mathbf{u}$ & $\mathbf{t}$ \\
$\sigma_{4}(a)$ & $\mathbf{t}$ & $\mathbf{u}$ & $\mathbf{f}$ & $\mathbf{t}$ \\
Danger & $\mathbf{t}$ & $\mathbf{f}$ & $\mathbf{i}$ & $\mathbf{u}$ \\
\hline
\end{tabular}

Membership of $a$ in the lower approximation of Danger for information source $I_{1}$, is obtained by computing $\forall y \in U\left[y \in \sigma_{1}(a) \hookrightarrow y \in\right.$ Danger $]$.

$$
\begin{aligned}
a \in \text { Danger }_{\sigma_{1}}^{+}= & \operatorname{GLB}_{y \in U}^{t}\left\{y \in \sigma_{1}(a) \hookrightarrow y \in \text { Danger }\right\}= \\
& \operatorname{GLB}^{t}\{\mathbf{t} \hookrightarrow \mathbf{t}, \mathbf{i} \hookrightarrow \mathbf{f}, \mathbf{u} \hookrightarrow \mathbf{i}, \mathbf{f} \hookrightarrow \mathbf{u}\}=\operatorname{GLB}^{t}\{\mathbf{t}, \mathbf{i}, \mathbf{i}, \mathbf{t}\}=\mathbf{i}
\end{aligned}
$$

Membership of $a$ in the upper approximation of Danger for information source $I_{1}$, is obtained by computing $\exists y \in U\left[y \in \sigma_{1}(a) \wedge_{t} y \in\right.$ Danger $]$.

$$
\begin{aligned}
a \epsilon \text { Danger }_{\sigma_{1}}^{\oplus}= & \operatorname{LUB}_{y \in U}^{t}\left\{y \in \sigma_{1}(a) \wedge_{t} y \epsilon \text { Danger }\right\}= \\
& \operatorname{LUB}^{t}\left\{\mathbf{t} \wedge_{t} \mathbf{t}, \mathbf{i} \wedge_{t} \mathbf{f}, \mathbf{u} \wedge_{t} \mathbf{i}, \mathbf{f} \wedge_{t} \mathbf{u}\right\}=\operatorname{LUB}^{t}\{\mathbf{t}, \mathbf{f}, \mathbf{u}, \mathbf{f}\}=\mathbf{t}
\end{aligned}
$$


Membership of $a$ in the lower approximation of Danger for the other information sources, $I_{2}, I_{3}$, and, $I_{4}$, is shown below.

$$
\begin{aligned}
& a \epsilon \text { Danger }_{\sigma_{2}}^{+}=\operatorname{GLB}_{y \in U}{ }^{t}\left\{y \epsilon \sigma_{2}(a) \hookrightarrow y \epsilon \text { Danger }\right\}=\mathbf{t} \\
& a \epsilon \text { Danger }_{\sigma_{3}}^{+}=\operatorname{GLB}_{y \in U}{ }^{t}\left\{y \epsilon \sigma_{3}(a) \hookrightarrow y \epsilon \text { Danger }\right\}=\mathbf{f} \\
& a \epsilon \text { Danger } \sigma_{\sigma_{4}}^{+}=\underset{y \in U}{\operatorname{GLB}}\left\{y \epsilon \sigma_{4}(a) \hookrightarrow y \epsilon \text { Danger }\right\}=\mathbf{u} .
\end{aligned}
$$

For any of the information sources above, $a \in \operatorname{Danger}_{\sigma_{i}}^{\oplus}=\mathbf{t}$, with $i \in\{2,3,4\}$.

We may compare the conclusions obtained with different information sources about the level of danger of object $a$. For instance, with the information from $I_{2}$, we are more certain that object $a$ is dangerous than with the information provided by $I_{1}$ or $I_{4}$, since $a \epsilon$ Danger $_{\sigma_{1}}^{+}=\mathbf{i}$ and $a \epsilon$ Danger $_{\sigma_{4}}^{+}=\mathbf{u}$, while $a \epsilon$ Danger $_{\sigma_{2}}^{+}=\mathbf{t}$. However, all information sources indicate that object $a$ may be dangerous, since $a \epsilon \operatorname{Danger}_{\sigma_{i}}^{\oplus}=\mathbf{t}$, with $i \in\{1,2,3,4\}$. Note that the fact lower and upper approximations are four-valued sets, allow us a finer comparison of the degree of evidence each information source has to support its conclusions.

The discussion in the previous paragraph brings us to the problem of collecting and combining knowledge from different sources (agents), which is a relevant point from the practical point of view. Note that this aspect is work in progress and it is not formalized in the paper. However, we use this example to illustrate our point and the relevance of knowledge ordering, $\leq_{k}$, in this context. For example, according to sources $I_{2}$ and $I_{4}$, we can conclude that $a$ is surely dangerous, since

$$
\operatorname{LUB}^{k}\left\{a \in \text { Danger }_{\sigma_{2}}^{+}, a \in \text { Danger }_{\sigma_{4}}^{+}\right\}=\operatorname{LUB}^{k}\{\mathbf{t}, \mathbf{u}\}=\mathbf{t} .
$$

On the other hand, sources $I_{2}$ and $I_{3}$ provide a contradictory information about membership of $a$ in the lower approximation of Danger and accordingly,

$$
\operatorname{LUB}^{k}\left\{a \epsilon \text { Danger }_{\sigma_{2}}^{+}, a \in \text { Danger }_{\sigma_{3}}^{+}\right\}=\operatorname{LUB}^{k}\{\mathbf{t}, \mathbf{f}\}=\mathbf{i} \text {. }
$$

Similarly, fusing information from all sources or from sources including $I_{1}$ result in common knowledge being $\mathbf{i}$.

\section{Conclusions}

We introduced a notion of four-valued set to model situations where both knowledge about properties of objects and knowledge of similarity between objects can be incomplete or inconsistent. For modelling inclusion of four-valued sets, we proposed a new implication operator. We have shown how the similarity-based notions of lower- and upper approximation used in the usual rough-set framework can be extended in a natural way to our four-valued setting.

As future work, we plan to develop and to implement a language that allows users to define and reason about vague relations. Vague relations will be represented as fourvalued sets and underlying similarity relations will be used to build relation (set) approximations, as we discuss in the paper. A knowledge base of such relations can then be queried by applications. 


\section{References}

1. Andersson, R., Vitória, A., Małuszyński, J., Komorowski, J.: Rosy: A rough knowledge base system. In: Slezak, D., Jingtao Y., J., Peters, J. F., Ziarko, W., Hu, X. (eds.), RSFDGrC 2005. LNCS, vol. 3642, pp. 48-58. Springer (2005)

2. Arieli, O., Avron, A.: The value of the four values. Artificial Intelleligence, vol. 102 (1), pp. 97-141. (1998)

3. Banerjee, M.: Rough sets and three-valued łukasiewicz logic. Fundamenta Informaticae, vol. 32, pp. 213-220 (1997)

4. Belnap, N.: A useful four-valued logic. In: Eptein, G., Dunn, J. (eds.), Modern Uses of Many Valued Logic, pp. 8-37. Reidel (1977)

5. Bolc, L., Borowik, P.: Many-Valued Logics, 1. Theoretical Foundations. Springer, Berlin (1992)

6. Doherty, P., Łukaszewicz, W., Szałas, A.: Tolerance spaces and approximative representational structures. In: Günter, A., Kruse, R., Neumann, B. (eds.), Proc. 26th German Conf. on AI, KI'2003. LNAI, vol. 2821, pp. 475-489. Springer-Verlag (2003)

7. Doherty, P., Łukaszewicz, W., Szałas, A.: Communication between agents with heterogeneous perceptual capabilities. Journal of Information Fusion, vol. 8(1), pp. 56-69. Elsevier (2007)

8. Doherty, P., Łukaszewicz, W., Skowron, A., Szałas, A.: Knowledge Representation Techniques. A Rough Set Approach. Studies in Fuziness and Soft Computing, vol. 202. SpringerVerlag (2006)

9. Doherty, P., Szałas, A.: On the correspondence between approximations and similarity. In: Tsumoto, S., Slowinski, R., Komorowski, J., Grzymala-Busse, J. (eds.), Proceedings of 4th International Conference on Rough Sets and Current Trends in Computing, RSCTC'2004. LNAI, vol. 3066, pp. 143-152. Springer-Verlag (2004)

10. Doherty, P., Szałas, A.: A correspondence framework between three-valued logics and similarity-based approximate reasoning. Fundamenta Informaticae, vol.75 (1-4), pp. 179193, (2007)

11. Dubois, D., Prade, H.: Putting rough sets and fuzzy sets together. Intelligent Decision Support: Handbook of Applications and Advances of the Rough Sets Theory, pp. 203-232. Kluwer (1992)

12. Fitting, M.C.: Bilattices in logic programming. In: Epstein, G. (ed.), 20th International Symposium on Multiple-Valued Logic. IEEE CS Press, Los Alamitos, pp. 238-247 (1990)

13. Ginsberg, M.: Multivalued logics: a uniform approach to reasoning in AI. Computational Intelligence, vol. 4, pp. 256-316 (1988)

14. Maluszyński, J., Szałas, A., Vitória, A.: A four-valued logic for rough set-like approximate reasoning. In: Duntsch, I., Grzymala-Busse, J., Orlowska, E., Polkowski, L., (eds.), Transactions on Rough Sets, Commemorative Issue. LNCS, vol. 4374, pp. 176-190 (2007)

15. Pawlak, Z.: Rough Sets. Theoretical Aspects of Reasoning about Data. Kluwer Academic Publishers, Dordrecht (1991)

16. Rescher, N.: Many-Valued Logic. McGraw Hill, New York (1969)

17. Skowron, A., Stepaniuk, J.: Tolerance approximation spaces. Fundamenta Informaticae, vol. 27, pp. 245-253 (1996)

18. Słowiński, R., Vanderpooten, D.: A generalized definition of rough approximations based on similarity. IEEE Trans. on Data and Knowledge Engineering, vol. 12(2), pp. 331-336, (2000)

19. Vitória, A.: A framework for reasoning with rough sets. In: Peters, J. F., Skowron, A. (eds.), Transactions on Rough Sets IV. LNCS, vol. 3700, pp. 178-276, (2005) 